\title{
Presenting an algorithm for choosing an optimum local service based on the qualitative feature of combining
}

\author{
Aalia Hemmati, Sima Emadi \\ Computer Engineering department. Islamic Azad university of Meybod, Yazd, Iran \\ Email address: \\ Aaliahemmati64@gmail.com (A.Hemmati),au_emadi@yahoo.com (S. Emadi)
}

\section{To cite this article:}

Aalia Hemmati, Sima Emadi. Presenting an Algorithm for Choosing an Optimum Local Service Based on the Qualitative Feature of Combining. International Journal of Intelligent Information Systems. Special Issue: Research and Practices in Information Systems and Technologies in Developing Countries. Vol. 3, No. 6-1, 2014, pp. 56-60. doi: 10.11648/j.ijiis.s.2014030601.20

\begin{abstract}
The combination of web services is the result of complex and increasing needs of the users and disability of single web services in resolving the users' needs. One of the important challenges in the field of web 2.0 is the combination of web services based on their qualitative features. Since it is probable that there would be several different combinations of services for achieving a specific goal, choosing the service is based on some qualitative features like combining, availability, acceptability, service cost and security. One of the important issues is the quantitative survey of combining rate of the two services shared on the combination. So in this research, in the first stage, for measuring the combining rate, the effective factors on this feature would be surveyed. In the second stage, for choosing the optimum service based on the qualitative feature of combining, the local strategy is used. The proposed algorithm in local strategy selects services that their combining rate is more than a specific threshold. The implementations and analysis show that the proposed algorithm presents the optimum service in user's view with an acceptable combining capability. Also, the analysis of results and an evaluation with a case study show the optimized results of the local proposed algorithms compared to existing methods.
\end{abstract}

Keywords: Web Service, Qualitative Feature of Service, Combining

\section{Introduction}

Service-oriented systems have special importance because of the possibility of working in heterogeneous distributed environments. The users of such systems use the web services that provide system components. In some cases, the needs of the users are not met with the single web service. However, each combination of services is not always the best possible solution and some criteria and features of the new combined service such as the quality of the new service can be a criterion for choosing the appropriate service. In fact, one of the important challenges in the field of web services is the combination of web services considering their quality. The need to combine web services is the result of complex and increasing requirements of users and disability of web services in responding to the goals of the users. Since it is likely that there would be several different combinations of services in achieving to a specific goal, choosing the service is based on some qualitative features like combining, availability, acceptability, service cost and security. One of the important issues that has been noted less is the accuracy quantitative survey of combination rate of the two services shared on the combined platform.

This feature brings the rate of services' adaptation with each other for combining without causing a problem in the runtime. After that the user applies for the required service, the component identifies the basic services with the combination of which it can get to the desired service and search for them inside the service store. Because of the diversity of different producers and services, it's likely that several candidate services be found for each desired efficiency.

For choosing the optimum service, we can consider the optimization in two ways: local and global. According to the conditions of the issue, the local optimality is used for choosing the optimum service based on the quality rate of combining. In [1], a framework is presented for choosing the service in terms of qualitative features in which the combination rate of services is focused. In [10], the combining feature is surveyed absolutely or with the use of local strategy, in this way that the two services are combined with each other or not. In [10], the optimization of services' combining has been done based on the local and global algorithm 
combination without considering services' combining.

In this research, by surveying the available weaknesses in the presented method in [10], a more accurate amount is obtained for combining feature and the optimum local optimization algorithm and an optimum service based on the combining rate is selected. While the most similar tasks have been spent on surveying the only effective factors on combining and the different ways of choosing the optimized compound service as local or global without considering the combining rate of services. . Some existing methods have more focused on similarities of services' input and output for surveying the combining rate of the two services and in cases that the other effective factors on combining is surveyed, no metric has been presented for its measuring. The presented model in this article has used the local optimality in comparison with the existing method and is done dynamically and in phase. It has evaluated the more number of parameters including cost, performance time, acceptability, reliability and combining in comparison with the other methods. As it goes on, the structure of the article is in this way that in the second chapter the combining rate is measured. In the third chapter, the proposed algorithm based on the qualitative feature of services' combining is presented. In the fourth chapter, simulation and results' analysis are stated and in the fifth chapter conclusion and future activities are proposed.

\section{Measuring the Qualitative Feature of Combining}

In this article, web service is considered as a black box and there is access only to its interface. And when the service is implemented as a web service, WSDL descriptions are the most common documents of service description. Since the available web services use WSDL, a semantic description in OWL-S language has been added to these services. OWL-S description of service is in three part of service profile, service model and service support [10].

Here, service profile part that includes input, output, name and service description and service support that includes transfer protocol and web service address are considered more. Also, it is supposed that services based on SOAP protocol communicate with each other [10].

The stages of measuring the qualitative feature of combining is in this way that in the first stage, the effective criteria on services' combining are surveyed in atomic terms. In the second stage, metric is obtained for the effective criteria. In the third stage, an appropriate weight for each criterion is obtained and their relationships with each other are determined. And finally, a metric for measuring the combining feature of services is presented.

Effective factors on combining are in two groups. One of them are the factors that are obtained of the communication between the desired candidate service with the previous existing service of the combination, like the surveying parameters of the similarity level of input- output parameters, effects and preconditions and the other are the factors surveyed on candidate atomic service. Table 1 is retrieved of [10] and shows the effective factors on combining in atomic terms with this difference that the importance level and the two qualitative parameters of reusability and adaptability is added in order that a more accurate rate of combining would be obtained.

Table 1. Effective criteria for qualitative feature of combining and metrics.

\begin{tabular}{|c|c|c|}
\hline Effective factors & Metric & _importance level \\
\hline Reusability [7] & $\lambda_{1}\left(S_{1}\right)=-0.5 *$ Coupling $+0.61 *$ ServiceGranularity $+0.61 *$ Parameter Granularity & Medium \\
\hline Adaptability[6] & $\lambda_{2}\left(\mathrm{~S}_{1}\right)=\mathrm{Num}_{\text {Consumers Satisfied Variants }} / \mathrm{Num}_{\text {TotalApplicableConsumers }}$ & High \\
\hline Granularity[5] & $\lambda_{3}\left(S_{1}\right)=1 /$ number of "atomic process" in owls file & \\
\hline Availability [4] & $\lambda_{4}\left(\mathrm{~S}_{1}\right)=\mathrm{WSOT} /(\mathrm{WSOT}+\mathrm{WSRT})$ & \\
\hline Loosely-Coupled [9] & $\begin{array}{l}\lambda_{6}\left(\mathrm{~S}_{1}\right)=1 /\left(\alpha_{\mathrm{x}}: \text { number of Complex data types }+\beta_{\mathrm{x}} \text { Number of service calls }\right) \\
\lambda_{6}\left(\mathrm{~S}_{1}\right)=\sum_{i=0}^{4} X i \\
\mathrm{x}_{\mathrm{i}} \subseteq\{0,0.2\}\end{array}$ & Medium \\
\hline Well-defined Interfaces [10] & $\begin{array}{l}\mathrm{xi}=\left\{\begin{array}{c}0.2, \text { hasparameter } \\
0, \text { no parameter }\end{array}\right. \\
\mathrm{x}_{0}=\text { input, } \mathrm{x}_{1}=\text { output }, \mathrm{x}_{2}=\text { precondition, } \mathrm{x}_{3}=\text { result, } \mathrm{x}_{4}=\text { category }\end{array}$ & High \\
\hline Study of Quality of Service [10] & $\lambda_{7}\left(\mathrm{~S}_{1}\right)=\left(\sum_{Q i \in \text { neg }} W_{i} \frac{Q_{i}^{\max }-Q_{i}}{Q_{i}^{\text {max }}-Q_{i}^{\min }}+\sum_{Q i \in \text { os }} W_{i} \frac{Q_{i}-Q_{i}^{\min }}{Q_{i}^{\max }-Q_{i}^{\min }}\right)$ & \\
\hline
\end{tabular}

\subsection{Metric for Measuring the Combining Feature}

This metric is presented for measuring the combining rate of the two continuous service that are combined with each other. The input of the issue is a workflow including some duties that are to be performed by the real services. These services are shown as $\mathrm{S}_{1} \mathrm{~S}_{2}$.

Assume that for the duty $\mathrm{x}_{1}$ a real service called $\mathrm{s}_{1}$ has been found. Now, the issue is that among the candidate services for the duty $x_{2}$, which real service is better to be selected. The choosing criterion is the service that would have a higher combining capability with the real service $s_{1}$. This metric is computed by relation 1 [10]:

$$
\begin{gathered}
\text { Composability }\left(\mathrm{S}_{1}\right)=\lambda\left(\mathrm{S}_{1}\right) \\
\lambda\left(\mathrm{S}_{1}\right)=\left\{\begin{array}{c}
0, \text { if } \prod_{i} \lambda_{\mathrm{i}}\left(\mathrm{S}_{1}\right)=0 \\
\sum a_{i} \lambda_{\mathrm{i}}\left(\mathrm{S}_{1}\right), \text { if } \prod_{\mathrm{i}} \lambda_{\mathrm{i}}\left(\mathrm{S}_{1}\right) \neq 0
\end{array}\right.
\end{gathered}
$$


In which, $I$ is the number of effective factors, $i=\{1 \ldots 7\}, a_{i}$ is the weight allocated to each criteria (between 0 and 1) $\sum_{i=1}^{n} a i=7$.

Finally, the obtained amount for combining is divided into the number of effective factors in formula so that the obtained number be normal. $\lambda_{\mathrm{i}}\left(\mathrm{S}_{1)}\right.$ shows the amount of each mentioned parameters for service combining. $\lambda\left(S_{1}\right) \in[0,1]$ is the combining rate of the desired candidate service. In order to normalize the acquired amount for combining, $\lambda\left(\mathrm{S}_{1)}\right.$ is divided into $\sum_{\mathrm{i}=1}^{\mathrm{n}}$ ai.

And finally, from among the candidate services, the service is selected that the combining rate of it be more than the defined threshold for combining. The combining threshold is the minimum amount that a service must have in order that face no problem in runtime when combining with other services. In this research, the combining threshold has determined 0.6 in the normal situation.

\section{The Proposed Model}

Optimizing can be considered in two local and global ways. In local optimality, for each action, a service of the highest quality from among the whole candidate services is chosen. Therefore, it is performed very fast but it doesn't provide a warranty for presenting a general optimum compound service. In global optimality, the services are chosen for each action that of their putting together the quality of compound service has the highest amount [10]. In proposed algorithm the local optimality is used for choosing the service based on the combining feature. It's needed to be noted that the algorithm does not operate voraciously because in global algorithm, several candidate service is needed for each action with the combining rate higher than the threshold in order that the general optimum compound service be presented in user's view. As it goes on, we state the details of this algorithm.

\section{Proposed Local Algorithm}

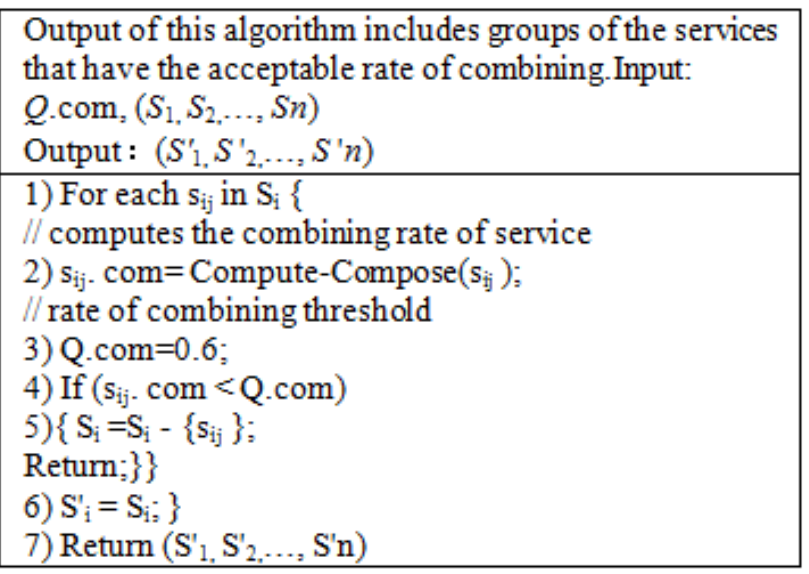

Figure1. Local strategy.

In local algorithm, the base of the task is the rate of the combining feature which is itself the combination of several other qualitative features like availability, cost, runtime, etc.
Therefore, it is necessary that the combining rate of the single existing based services be surveyed. When choosing the services from among the different services, the state is chosen that has an acceptable rate of combining and finally, leads to an optimized compound service. Stages of the proposed local algorithm according to the code-alike of Table 1 are as follows:

1. Environment of the system is determined. When a compound workflow enters the system, it is assumed that in the services' store, as for each existing action in workflow, there is a group of candidate services which perform that action.

2. The algorithm computes the combining rate of each candidate service for each action.

3. Rate of combining threshold would be considered 0.6 and combining rate of candidate services would be compared to the threshold amount.

4. From each group, the services which have the combining rate of less than the amount of threshold are deleted and the related group becomes updated.

5. The outputs of this algorithm are the groups of the services that have an acceptable rate of combining.

\section{Evaluation and Analysis of the Results}

The case study is considered as a way for evaluating the presented metric. In this regard, a real sample of the compound services is shown. A case study on "reserving a hotel" scenario was performed and the possibility of computing the presented formula for combining is surveyed. Finally, the accuracy of the function of the presented method was evaluated and compared with a valid method. Also, in the local algorithm, the time is spent on choosing the optimum service and the comparison of it with the local algorithm of the valid method and there are comparisons in this field with the existing methods. Stages of reserving a hotel are shown in Figure 2 in the form of a workflow.

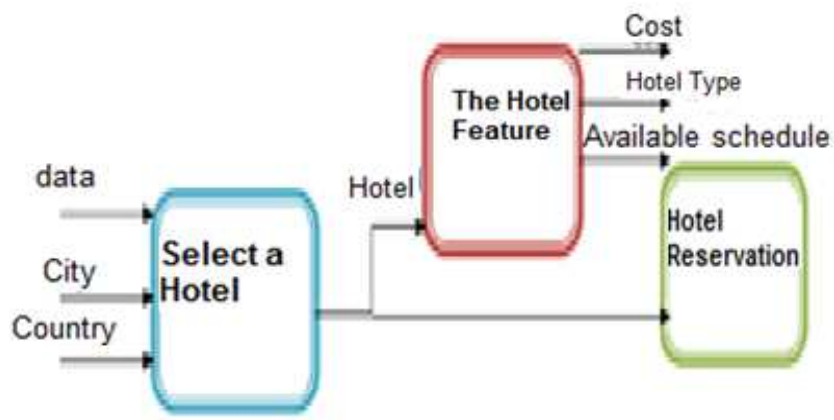

Figure 2. Reserving a hotel Scenario [10].

For adapting the mentioned duties in the input workflow with real services, the stores were searched in order to find the services that did each of these functions. Finally, for each of these duties, some candidate services were found that performed the function. The user requests that discovered services for doing these duties had the following qualitative features, as in Table 2. 
Table 2. Qualitative features [1]

\begin{tabular}{ll}
\hline Qualitative features & Renges \\
\hline Execution time & $0<$ exe $<10$ s \\
Reliability & $90<\mathrm{rel}<100$ \\
Reputation & $70<\mathrm{rep}<100$ \\
Price & $0<\mathrm{p}<1000 \$$ \\
\hline
\end{tabular}

In this research, the real existing services in method [10] that were taken from the service store sws tc were used for doing the case study in order for the results to be closer to reality. Finally, the results were compared with method [10].

\subsection{Comparing the Combining Rate of Candidate Services in Atomic Terms}

Computing the combining rate of discovered services for the task "choosing a hotel" is given in Table 3. All the effective criteria on combining is surveyed and computed. Finally the combining rate of each service for "choosing a hotel" is obtained according to relation 1 .

Table 3. Computing the combining rate of services for the task "reserving a hotel"

\begin{tabular}{|c|c|c|c|c|c|c|c|c|}
\hline Services & $\lambda_{1}($ gran$)$ & $\lambda_{2}$ (ava) & $\lambda_{3}(\operatorname{cou})$ & $\lambda_{4}($ reus $)$ & $\lambda_{5}$ (adap) & $\lambda_{6}$ (inte) & $\lambda_{7}(\mathrm{qos})$ & Com \\
\hline WorldwideHotel InfoService & 1 & 0.75 & 1.5 & 0.35 & 1 & 0.4 & 0.32 & 0.76 \\
\hline CityCountryHotel Service & 1 & 0.97 & 1.7 & 0.25 & 1 & 0.6 & 0.36 & 0.84 \\
\hline CityHotelService & 1 & 0.9 & 1.6 & 0.3 & 1 & 0.4 & 0.225 & 0.77 \\
\hline Cityhotels & 1 & 0.85 & 1.2 & 0.5 & 1 & 0.6 & 0.154 & 0.75 \\
\hline
\end{tabular}

\subsection{Comparing the Function of Proposed Metric with the Existing Method Based on Combining Rate}

In this chapter, we spend time on comparing the obtained amounts for combining in the proposed method with method [10] in atomic terms. Table 3 shows the obtained combining rate in both methods for the task "choosing a hotel".

Table 4. Comparing the combining rate of services for the task "reserving a hotel" in both methods.

\begin{tabular}{lll}
\hline Candidate services & combining rate in offered method & combining rate in presented method[10] \\
\hline WorldwideHotel InfoService & 0.76 & 0 \\
CityCountaryHotelService & 0.84 & 0.68 \\
CityHotelService & 0.77 & 0.57 \\
CityHotels & 0.75 & 0.63 \\
\hline
\end{tabular}

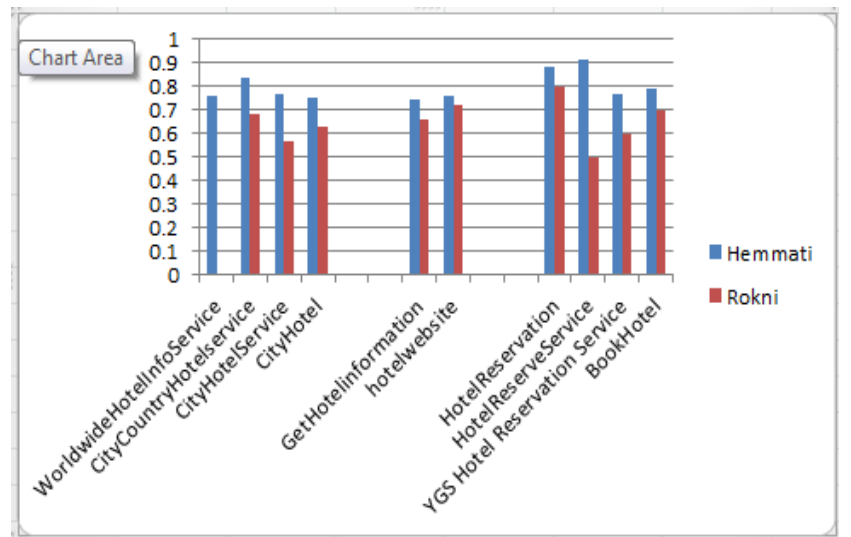

Figure 3. Comparing the obtained combining rate in the proposed method and presented method in [10].

The chart in Figure 3 shows a comparison between the obtained combining rate in the proposed method and presented method in [10]. As seen in Figure 3, in the presented method, by adding the two effective factors of "reusability" and "adaptability", a more accurate rate was obtained for combining feature of candidate services in comparison to method [10].

The chart in Figure 3 shows a comparison between the obtained combining rate in the proposed method and presented method in [10]. As seen in Figure 3, in the presented method, by adding the two effective factors of "reusability" and "adaptability", a more accurate rate was obtained for combining feature of candidate services in comparison to method [10].

\subsection{Comparing the Proposed Local Algorithm with the Local Algorithm of Existing Method Based on the Combining Rate}

As it was stated before, according to the proposed local algorithm, from among the candidate services for each action, the service which has the combining rate of more than the threshold would be selected as the optimum service but in method [10], optimality of choosing the service is done only locally and voraciously and the service is selected as the optimum service that has a higher rate of combining. The chart in Figure 3 demonstrates a comparison between the proposed local algorithm output and the method presented in [10].

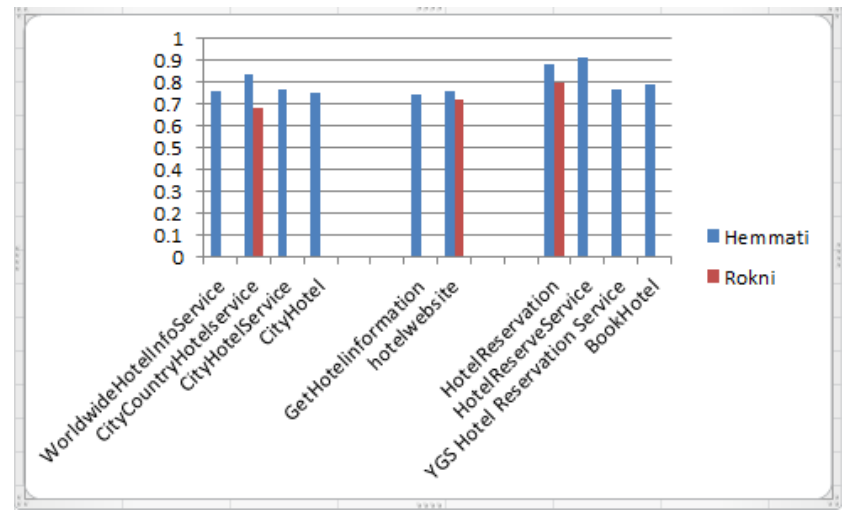

Figure 4. Comparing the proposed local algorithm's output and the presented method in [1].

As observed in the chart of Figure 4, in method [10], 
because of performing voraciously, for the task of choosing hotel, the service "CityCountryHotelservice", for the task of surveying hotel information, the service "hotelwebsite" and for the task reserving a hotel, the service "HotelReservation" is selected. Also, because of performing voraciously, a service is likely to be selected that, after combining with other selected services, cannot finally obviate users' needs. In the proposed local method, a service choosing was done based on an acceptable rate of combining in order that, in the implementation time for combining with other services, it did not get into trouble. In this case study, all the candidate services obtained the acceptable rate of combining and were selected.

\section{Discussion, Conclusion and Future Works}

In this article, by surveying the existing weaknesses in method [10] and the more number of effective factors on the combining feature, a more accurate amount is obtained. Then, a local algorithm based on the combining rate of the services is presented for optimality of choosing services locally. This algorithm was designed in such a way that can be combined with global algorithm in future and finally a general optimized compound service was presented. While in method [10], in local optimality, because of performing voraciously, presenting a general optimized compound service in user's view wan not warrantied. Most of the similar tasks survey the only effective factors on combining and no metric is presented for them. Also, some different methods have spent time on choosing the optimized compound service locally or globally without considering the combining rate of the services. In line with this research, we can evaluate the other qualitative parameters in addition to the combining rate in future and present a metric for it. A more accurate surveying on weights allocated to each of combining parameters is another job that can be surveyed in future.

\section{References}

[1] Q. Yu, M. Rege, A. Bouguettaya,B. Medjahed, M. Quzzani, A Two-Phase Framework For Quality-Aware Web Service Selection, Service Oriented Computing and Applications Journal, Vol 4, No.2, pp. 63-79, 2010.

[2] M., M., Akbar, M., S., Rahman, M., Kaykobad, E., G, Manninga, G., C., Hoja, Solving the Multidimensional Multiple-choice Knapsack Problem by Constructing Convex Hulls, Computers \& Operations Research Journal, Vol. 33, pp. 1259- 1273, 2006.

[3] S. Choi, S. Jin Sun and S. D. Kim, QoS Metrics for Evaluating Services from the Perspective of Service Providers, in IEEE International Conference, e-Business Engineering, ICEBE (2007), pp. 622-625, 2007.

[4] J. Fang, S. Hu and Y. Han, A Service Interoperability Assessment Model for Service Composition, in Proceedings of the 2004 IEEE International Conference on Services Computing, IEEE Computer Society, pp. 153-158, 2004.

[5] R. Sindhgatta, B. Sengupta and K. Ponnalagu, Measuring the Quality of Service Oriented Design, in Proceedings of the 7th International Joint Conference on Service-Oriented Computing, Springer-Verlag: Stockholm, pp. 485-499, 2009.

[6] S. Choi and S. Kim, A Quality Model for Evaluating Reusability of services in SOA, 10th IEEE Conference on E-commerce Technology and the Fifth IEEE Conference on Enterprise Computing, E-Commerce and EServices, 2008.

[7] B. Shim, S. Choue, S. Kim and S .Park, A Design Quality Model for Service Oriented Architecture, 15th Asia Pacific Software Engineering Conference, pp.403-410, 2008.

[8] G. Feuerlicht, Design of Composable Services, in Service-Oriented Computing, ICSOC Workshops, 2009.

[9] N.M. josuttis, SOA in practice the Art of distributed system design, oreilly: united state. pp. 35-46, 2007.

[10] Z. Rokni, " choosing the service in service-based architecture based on qualitative features" MA thesis, Shahid Beheshti university, 2011 